\title{
PAUTAS PARA EL DIAGNÓSTICO DE LA DIABETES MELLITUS GESTACIONAL
}

\author{
Gloria Larrabure Torrealva ${ }^{1}$
}

\begin{abstract}
RESUMEN
La diabetes gestacional es la alteración del metabolismo de los hidratos de carbono, de severidad variable, que comienza o se reconoce por primera vez durante el embarazo.

La búsqueda de diabetes en el embarazo debe hacerse desde el primer nivel de atención, en toda embarazada, presente o no factores de riesgo, de acuerdo a los recursos disponibles y es conveniente realizarla el tamizaje mediante una Prueba de Tolerancia Oral a la Glucosa (PTOG) a las 24-28 semanas de gestación.
\end{abstract}

Palabras clave: Diabetes gestacional; Glucosa; Prueba de Tolerancia Oral a la Glucosa (fuente: DeCS BIREME).

\section{GUIDELINES FOR THE DIAGNOSIS OF GESTATIONAL DIABETES MELLITUS}

\author{
ABSTRACT \\ Gestational diabetes is the impaired metabolism of carbohydrates, of varying severity that begins or is first recognized \\ during pregnancy. \\ The search for diabetes in pregnancy should be done from the first level of care throughout pregnancy, present or no risk \\ factors, according to available resources and should be done by the screening using a test Oral Glucose Tolerance (OGTT \\ ) at 24-28 weeks gestation. \\ Key words: Gestational Diabetes; Glucose; Test Oral Glucose Tolerance (source: MeSH NLM)..
}

\section{INTRODUCCIÓN}

A la fecha, no existen normas internacionales uniformes para la determinación y el diagnóstico de Diabetes Gestacional, existiendo factores de confusión (incluyendo la obesidad, la edad materna avanzada o las complicaciones médicas asociadas).

Dependiendo de la población estudiada, la prevalencia de Diabetes Mellitus Gestacional (DMG), en América Latina y el Caribe varía entre el 1 y el $14 \%$ de los embarazos. La DMG continúa siendo un importante problema en salud pública y se espera que el número de mujeres afectadas aumente como consecuencia de los hábitos alimentarios (dietas hipercalóricas) y el sedentarismo.

En 1964, O’Sullivan JB, Mahan C, definió la diabetes gestacional como "la alteración del metabolismo de los hidratos de carbono (intolerancia a los carbohidratos), de severidad variable, que comienza o se reconoce por primera vez durante el embarazo" y que se aplica independientemente de si se requiere o no de insulina o si la alteración persiste después del embarazo. No excluía la posibilidad que la alteración metabólica reconocida hubiere estado presente antes de la gestación determinando el riesgo para desarrollar Diabetes Mellitus 2 tras el embarazo, sin identificar los resultados perinatales adversos en gestantes con hiperglicemia.

\section{CRITERIOS DIAGNÓSTICOS}

La Asociación Americana de Diabetes (ADA) recomendaba el diagnóstico de la DMG con una prueba en 2 pasos (prueba de $50 \mathrm{~g}$ y $100 \mathrm{~g}$ ) haciendo un tamizaje o screening para seleccionar a la población susceptible de desarrollar DMG, basándose en criterios de riesgo, con una asociación positiva con la prevalencia de DMG: alto riesgo: 5,12 \%; bajo riesgo: 0,59 \%; recomendando:

- En pacientes con bajo riesgo: no hacer tamizaje ni Prueba de Tolerancia Oral a la Glucosa (PTOG).

- En pacientes con riesgo intermedio: hacer el tamizaje entre las 24 y 28 semanas

- En pacientes con alto riesgo: realizar directamente la PTOG en la 1ra. consulta en el primer trimestre del embarazo y si es posible practicar una $\mathrm{HbA} 1 \mathrm{c}$ y argumentando que "El tamizaje no es costo-efectivo en pacientes que cumplan con todos los criterios de bajo riesgo"

Sin embargo, el diagnóstico selectivo por riesgo materno excluye a un 10 a $40 \%$ de la población y pierde entre un 3 a $10 \%$ de los diagnósticos positivos.

Es por ello que la International Association of Diabetes and Pregnancy Study Groups (IADPSG), postuló que todas las mujeres sin factores de riesgo deberían tener una PTOG con

Médico Endocrinóloga del Instituto Nacional Materno Perinatal . Presidenta del Consenso Peruano de Diabetes Gestacional. Miembro del grupo DIAMU ( Diabetes en la Mujer- GLED) 
$75 \mathrm{~g}$ a las 24-28 semanas de gestación desarrollando puntos de corte para las glicemias a la hora y dos horas en relación con las complicaciones del embarazo, tras un ayuno de al menos 8 horas, siendo los valores normales para la PTOG:

- Basal: $92 \mathrm{mg} / \mathrm{dl}(5,1 \mathrm{mmol} / \mathrm{l})$,

- $1^{\circ}$ hora: $180 \mathrm{mg} / \mathrm{dl}(10,0 \mathrm{mmol} / \mathrm{l})$,

- $2^{\circ}$ hora: $153 \mathrm{mg} / \mathrm{dl}(8,5 \mathrm{mmol} / \mathrm{l}$.

\section{* Un solo valor anormal hace el diagnóstico de diabetes} gestacional

En Mayo del 2008, el estudio: Hyperglycemia and Adverse Pregnancy Outcomes, (Estudio HAPO), planteó que tales criterios diagnósticos duplicarían la prevalencia actual de DMG (17.8\%), que la diabetes gestacional aumenta un 1,75 veces el riesgo de tener un recién nacido grande para la edad gestacional y que la obesidad es un fuerte predictor de DMG. Se observó una fuerte asociación, en relación linear, tanto para los eventos adversos primarios:peso al nacer por encima del percentil 90 para la edad gestacional, parto por cesárea primaria, hipoglicemia clínica neonatal, Péptido $\mathrm{C}$ en sangre de cordón por encima del percentil 90 (hiperinsulinemia fetal), como secundarios: parto prematuro (antes de las 37 semanas de gestación), distocia de hombros, lesión en el parto, necesidad de cuidados intensivos neonatales, hiperbilirrubinemia, preeclampsia, existiendo una relación directamente proporcional entre niveles de glicemia y resultados perinatales sin umbrales de corte para el aumento de riesgo

En el año 2011, el ADA, postuló:

"Si se diagnostica DM2 en el primer trimestre de la gestación, es muy probable que ésta sea una diabetes mellitus pre gestacional, no diagnosticada antes de la gestación: diabetes manifiesta", proponiéndose una nueva terminología:

- Diabetes Manifiesta (Overt Diabetes)

- Diabetes Gestacional

Esta nueva estrategia diagnóstica si bien aumentará sustancialmente la prevalencia de desórdenes hiperglicémicos en el embarazo, es consistente con el aumento de la prevalencia de obesidad y desórdenes metabólicos en la población de adultos jóvenes y con los reportes recientes de aumento en la prevalencia de DMG y DM pre existente en mujeres embarazadas". Los nuevos criterios ayudan a uniformizar criterios a nivel mundial, para comparación de trabajo futuros. "La hiperglicemia materna, menos severa que la diabetes, está relacionada con importantes alteraciones perinatales y sus efectos pueden ser disminuidos con el tratamiento adecuado, aunque el punto para iniciar tratamiento aún no está establecido".

En Latinoamérica, el grupo DIAMU: "Diabetes en la Mujer", perteneciente al grupo Latinoamericano de Epidemiología en Diabetes,(GLED), reconocido por la
IDF, acordó en Buenos Aires en Noviembre 2011 que se debe hacer búsqueda de Diabetes en el embarazo en toda embarazada, presente o no factores de riesgo, de acuerdo a los recursos disponibles y determinó los factores de riesgo para diabetes gestacional en población de Latinoamérica, siendo éstos:

- Antecedente de diabetes gestacional en embarazo anterior

- Edad mayor o igual a 30 años.

- Antecedentes de diabetes en familiares de $1^{\circ}$ y $2^{\circ}$ grado

- Pacientes con índice de masa corporal de 27 o más al comienzo del embarazo.

- Antecedentes de macrosomía fetal al nacer (un hijo de 4000 gr o más), o alto peso para la edad gestacional.

- Glicemia en ayunas mayor de $85 \mathrm{mg} / \mathrm{dl}$.

- Síndrome de poliquistosis ovárica.

- Antecedentes de mortalidad perinatal inexplicada.

- Alto o bajo peso al nacer de la madre.

- Antecedentes de preeclampsia.

Plantea también que sería conveniente solicitar anticuerpos anti GAD a pacientes con diagnóstico de DIABETES, sin factores de riesgo, cuando presenten 20 más de las siguientes características:

- Menores de 25 años.

- Sin antecedentes familiares de diabetes.

- Normo o bajo peso.

- Con glicemia en ayunas alteradas antes de la semana 20 de gestación

\section{CONCLUSIONES}

A) ¿Cuál sería el criterio diagnóstico de diabetes gestacional en nuestras gestantes?

La búsqueda de Diabetes en el Embarazo debe hacerse desde el primer nivel de atención, en toda embarazada, presente o no factores de riesgo, de acuerdo a los recursos disponibles y es imprescindible realizarla en todas las embarazadas que presenten factores de riesgo para diabetes en el embarazo

- Se deberá practicar PTOG a todas las gestantes en las semanas 24-28 del embarazo, tengan/no factores de riesgo (despistaje universal)

- Si las gestantes tienen factores de riesgo para diabetes gestacional, es conveniente practicar una PTOG lo más temprano en la gestación, en los lugares que cuenten con los recursos adecuados

- La Hb A1c no representa un test adecuado para detectar intolerancia a la glucosa con capacidad suficientemente discriminativa y sensible. El valor de 6,5\% representa uno de los criterios de diagnóstico de diabetes manifiesta en la gestación (IADPSG-ADA). 
- Es conveniente que toda gestante con diabetes gestacional, diagnosticada en el primer nivel de atención, sea transferida a un nivel de atención superior

El diagnóstico de Diabetes Gestacional se hace cuando la mujer gestante se presenta con alguno de los dos siguientes criterios:

a) La glicemia de ayuno iguala o supera el valor de $92 \mathrm{mg} / \mathrm{dL}(5,1 \mathrm{mmol} /)$, Y es inferior a $126 \mathrm{mg} / \mathrm{dL}$ en cualquier momento de la gestación

b) En las semanas 24-28 del embarazo, la PTOG (75 g) muestra al menos un resultado anormal: glicemia de ayuno, igual o superior a $92 \mathrm{mg} / \mathrm{dL}$ $(5,1 \mathrm{mmol} / \mathrm{L})$, aunque inferior a $126 \mathrm{mg} / \mathrm{dL}(7,0$ mmolL); glicemia a la hora, igual o superior a 180 $\mathrm{mg} / \mathrm{dL}$ (10,0 mmol/L); glicemia a las 2 horas, igual o superior a $153 \mathrm{mg} / \mathrm{dL}$ (8,5 mmol/L).

(La glicemia de ayuno igual o mayor de $126 \mathrm{mg} / \mathrm{dL}$, es sugestiva de una diabetes manifiesta o diabetes mellitus pregestacional)

B) ¿Cuáles son los parámetros de control glicémico en pacientes con diabetes gestacional?

- Glicemia en ayunas: 70 a 90 mg/dl.

- Glicemia 1 hs postprandial: 90 a 140 mg/dl medida a partir del inicio a la comida.

- Glicemia 2 hs postprandial: 70 a $120 \mathrm{mg} / \mathrm{dl}$ medida a partir del inicio a la comida.

Estos parámetros deberán acompañarse de Cetonuria Negativa

En el primer control pre- natal, se deberá Individualizar un plan de ganancia de peso adecuado para cada gestante

Ganancia de peso adecuada durante la gestación según el Instituto de Medicina 2009 (USA)

\section{- Embarazo único:}

Peso antes del embarazo

Ganancia de peso recomendada

Bajo peso (IMC inferior a 18,5)

28 a 40 libras (unos 13 a 18 kilogramos)

Peso normal (IMC de 18,5 a 24,9)

25 a 35 libras (unos 11 a 16 kilogramos)

Sobrepeso (IMC de 25 a 29,9)

15 a 25 libras (alrededor de 7 a 11 kilogramos) Obesa (IMC de 30 o más) 11 a 20 libras (alrededor de 5 a 9 kilogramos)

\section{- Embarazo gemelar:}

Peso antes del embarazo

Ganancia de peso recomendada
Peso normal (IMC de 18,5 a 24,9)

37

a 54 libras (alrededor de 17 a 25 kilogramos)

Sobrepeso (IMC de 25 a 29,9)

a 50 libras (unos 14 a 23 kilogramos)

Obesa (IMC de 30 o más)

a 42 libras (unos 11 a 19 kilogramos)

Los cálculos asumen una ganancia de peso de 0,5$2 \mathrm{~kg}(1,1-4,4 \mathrm{lbs})$ en el primer trimestre (Siega-Riz et al, 1994; Abrams et al, 1995; Carmichael et al, 1997)

C) ¿Se debe usar metformina u otros hipoglicemiantes orales durante la gestación?

Puede usarse metformina en la gestación en las gestantes con antecedentes de insulinorresistencia y ovario poliquístico quienes hayan estado en tratamiento previo, antes de la gestación con metformina y hayan gestado tras uso de ésta y quienes deberán firmar un consentimiento informado de aceptación de continuar con el tratamiento durante la gestación.

No se usará metformina de novo en la gestación.

\section{REFERENCIAS BIBLIOGRÁFICAS}

1. Proceedings of the 4th International Workshop Conference on GDM. Diabetes Care 1998; 21-Suppl 2:B1

2. Mauricio D, Corcoy R, Codina M, Balsells M, Puig-Domingo M, Pou JM, de Leiva A. Islet ceel antibodies identify a subset of gestational diabetic women with higher risk of developing diabetes mellitus short after pregnancy. Diabetes Nutr Metab 1992; 5:237

3. IADPSG. Recommendations on the Diagnosis and Classification of Hyperglycemia in Pregnancy. Diabetes Care 2010; 33:676

4. American Diabetes Association. Diagnosis and classification of diabetes mellitus. Diabetes Care 2011;34 (Suppl1):S62

5. HAPO Study Cooperative Research Group. Hyperglycemia and adverse pregnancy outcomes. N Engl J Med 2008; 358:1991

6. O'Sullivan JB. Subsequent morbidity among gestational diabetes women. In: Carbohydrate metabolism in pregnancy and the newborn. Sutherland, HPWP, Stowers JM (Eds), Churchill Livingstone, New York 1984, p.174.

7. Barahona JM, Sucunza N, García-patterson A, Hernández M, Adelantado JM, Ginovart G, de Leiva A, Corcoy R. Period of Gestational Diabetes Mellitus Diagnosis and maternal and fetal morbidity. Acta Obstet Gynecol Scand 2005; 84:622

8. Bevier WC, Fischer R, Jovanovic L. Tratment of women with an abnormal glucose challenge test (but a normal oral glucose tolerance test) decreases the prevalence of macrosomia. Am J Perinatol 1999; 16:269.

9. Crowther CA, Hiller JE, Moss JR et al. Effect of treatment of GDM on pregnancy outcomes. N Engl J Med 2005; 352:2477

10. Landon MB, Spong CY, Thorn E, et al. A multicenter, randomized trial of treatment for mild GDM. N Engl J Med 2009;361:1339.

11. Alwan N, Tuffnell DJ, West J. Treatment for GDM. Cochrane Database Syst Rev 2009:CD003395

12. Franz MJ, Bantle JP, Beebe CA, et al. Evidence-based 
nutrition principles and recommendations for the treatment and prevention of diabetes and related complications. Diabetes Care 2003; 26 (Suppl 1):S51.

13. American Diabetes Association. Gestational Diabetes Mellitus. Diabetes Care 2004; 27 (Suppl 1):S88.

14. Weisz B, Shrim A, Homko CJ et al. One hour versus two hours postprandial glucose measurement in GDM: a prospective study. J Perinatol 2005; 25:241.

15. Corcoy R, Codina M, Pérez A, de la Torre W, Urgelés JR, Pou JM, de Leiva A. Self-monitoring of blood glucose in women attending a diabetes and pregnancy clinic: accuracy in reporting glucose values and fulfilment of scheduled timing. Diabetes Nutr Metab 1991; 4:23

16. American College of Obstetricians and Gynecologists. GDM. ACOG practice bulletin $\mathrm{N}^{\circ} 30,2001$

17. Harmon KA, Gerard L, Jensen DR, et al. Continuous glucose profiles in obese and normal-weight pregnant women on a controlled diet: metabolic determinants of fetal growth. Diabetes Care 2011; 34:1660.

18. Nicholson W, Bolen S, Witkop CT, et al. benefits and risks of oral diabetes agents compared with insulin in women with GDM. Obstet Gynecol 2009; 113:193

19. Dhulkotia JS, Ola B, Fraser R, Farrell T. Oral hypoglycemic agents vs insulin in management of GDM : a systematic review and metaanalysis. Am J Obstet Gynecol 2010; 203:457.e1.

20. Langer O, Conway DL, Berkus MD, et al. A comparison of glyburide and insulin in women with GDM. N Engl J Med 2000; 343:1134

21. Conway DL, Gonzales O, Skiver D. Use of glyburide for the treatment of GDM: the San Antonio experience. J Matern Fetal Neonatal Med 2004; 15: 51.

22. Lain KY, Garabedian MJ, Daftary A, Jeyabalan A. Neonatal adiposity following maternal treatment of GDM with glyburide compared with insulin. Am J Obstet Gynecol 2009; 200:501.e1.

23. Rowan JA, hague WM, Gao W et al. Metformin vs insulin for the treatment of GDM. N Engl J Med 2008; 358:2003

24. Moore LE, Clokey D, Rappaport VJ, Curet LB. Metformin cpmpared with glyburide in gestational diabetes: a randomized controlled trial. Obstet Gynecol 2010; 115:55.
25. Ljäs $H$, Vääräsmaki $M$, Morin-Papunen $L$, et al. Metformin should be considered in the treatment of gestational diabetes : a prospective randomized study. BJOG 2011; 118:880

26. Vanky E, Zahlsen K, Spigset $O$, et al. Placental passage of metformin in women with polycystic ovary síndrome. Fertile Steril 2005; 83:15715

27. Rowan JA, Rush EC, Obolonkin V, et al. Metformin in gestational diabetes : the offspring follow-up (MIG TOFU): body composition at 2 years of age. Diabetes Care 2011; $34: 2279$

28. Vanky E, Stridsklev S, Heimstad R, et al. Metformin versus placebo from first trimester to delivery in polycystic ovary syndrome: a randomized controlled multicenter study. J Clin Endocrinol Metab 2010; 95:E448

29. Zucker P, Simon G. Prolonged symptomatic neonatal hypoglycemia associated with maternal chlorpropamide therapy. Pediatrics 1968; 42:84

30. Kemball ML, Mclver C, Milner RD, et al. neonatal hypoglycemia in infants of diabetic mothers given sulphonylurea drugs in pregnancy. Arch Dis Child 1970; 45:696

31. Chan LY, Yeung JH, Lau TK. Placental transfer of rosiglitazone in the first trimester of human pregnancy. Fertil Steril 2005; 83:955

32. Metzger B, Buchanan TA, Coustan D, de Leiva A, et al Summary and Recommendations of the Fifth International Work-shop-Conference on Gestational Diabetes Mellitus. Diabetes Care 2007; 30(Suppl 2):S251

33. Albareda M, Caballero A, Badell G, Piquer S, Ortiz A, de Leiva A, Corcoy R. Diabetes and abnormal glucose tolerance in women with previous gestational diabetes. Diabetes Care 2003; 26:1199.

34. Knowler WC, Barret-Connor E, Fowler SE, et al. Reduction of the incidence of type 2 diabetes with lifestyle intervention or metformin. N Engl J Med 2002;346:393

35. Ratner RE, Christophi CA, Metzger BE, et al. Prevention of diabetes in women with a history of gestational diabetes: effects of metformin and lifestyle interventions. J Clin Endocrinol Metab 2008; 93:4774. 